
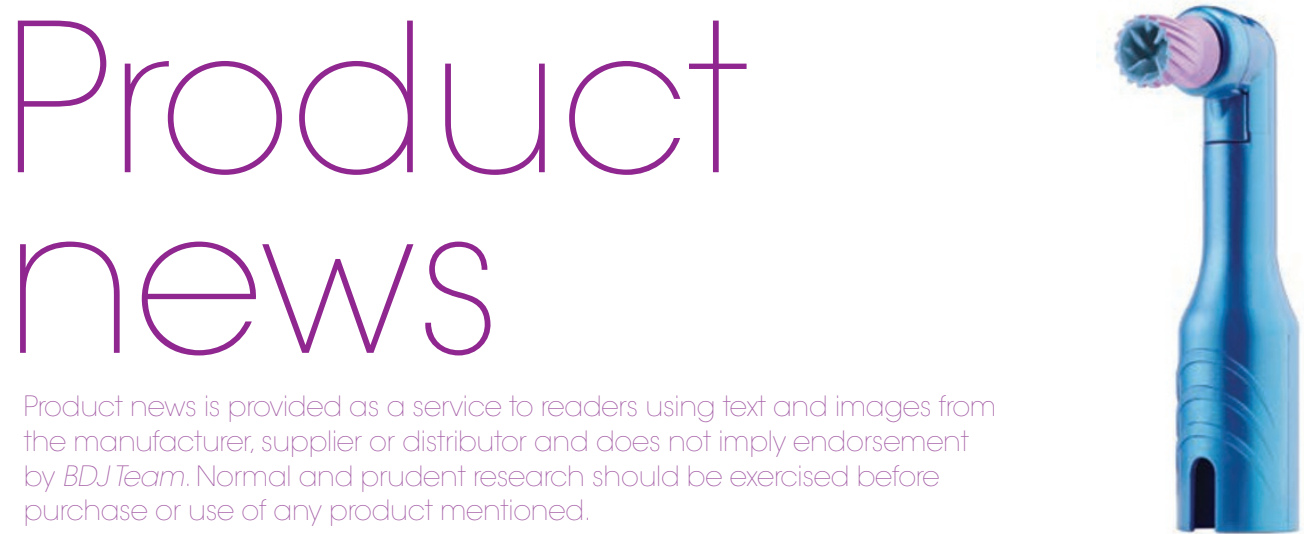

Product news is provided as a service to readers using text and images from the manufacturer, supplier or distributor and does not imply endorsement by BDJ Team. Normal and prudent research should be exercised before purchase or use of any product mentioned.

\section{CLEANING BETWEEN TEETH FOR OVER 200 YEARS}

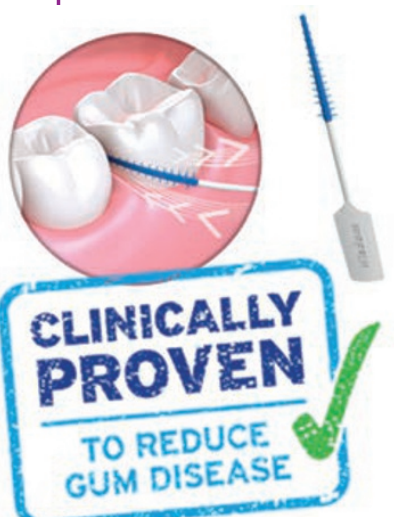

Do you

recommend the

Wisdom Clean

Between Rubber

Interdental

Brushes to your

patients?

Wisdom

Toothbrushes

has been a

trusted brand

for oral health

solutions for

more than 235 years. Among the highlights of its portfolio are the Wisdom Clean Between

Rubber Interdental Brushes, which are

clinically proven to reduce gingival disease.

Speak to the team at the BSDHT Oral

Health Conference and Exhibition to find out how the flexible, tapered brushes provide an effective yet comfortable interdental clean.
They'll show how the super soft micro-fine rubber filaments slide easily between the teeth and why the wire-free brushes are perfect for patients who have previously found wire interdental brushes difficult or uncomfortable to use.

You'll also discover the new Proflex Interdental Brushes with a unique, flexible neck and curved handle. Improving access to all areas of the mouth while reducing pressure on the wire for extended product life, these brushes ensure a comfortable patient experience.

Plus, don't miss the opportunity to find out more about the Wisdom Super Slim Interspace Brush, Wisdom Clean Between Easy Slide Y Shaped Floss with PTFE tape, the Wisdom Interproximal Brush.

Visit www.wisdomtoothbrushes.com or call 01440714800. Wisdom Easy Flosser with PTFE tape and the

\title{
CREATE AN EXCEPTIONALLY DRY SALIVA FIELD
}

We all want our patients to feel comfortable and secure when they enter our practice. The real test of ensuring their comfort comes when they take their seat in the dental chair.

In addressing the issue of saliva control during treatment, NeoDrys Saliva Absorbents ensure a more relaxing environment for your patient and an exceptional working area for you. Say goodbye to awkward fitting cotton absorbents; the super absorbent core creates an exceptionally dry field and protects from parotid saliva contamination with control of up to 15 minutes.

NeoDrys Saliva Absorbents, available exclusively from Dental Express, a trading division of Surgery Express LLP, are also ideal to treat patients who find dental treatment uncomfortable because of a pronounced gag reflex.

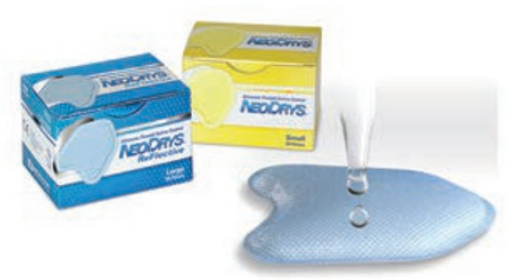

Available in large and small sizes, NeoDrys Saliva Absorbents are also available with reflective backing to assist you in your intraoral procedures. There is no danger of distracting leakage during treatment.

Take saliva control in hand with NeoDrys Saliva Absorbents, and discover why it outperforms all other absorption products on the market. Visit www.dental-express.co.uk or call 08007076212 disposable prophy

angles as well as pastes for polishing and the removal of discolorations. They are so effective thanks to the remarkable efficiency of baking powder, an excellent brightening effect, a neutral $\mathrm{pH}$ value and they are less abrasive than comparable products.

The 'Mint' and 'Berry' flavoured pastes are vegan, as well as sugar and gluten free. They are available in colourful single doses and are therefore easy to identify. The accompanying, sterilisable holder simplifies holding the paste while polishing.

Disposable Prophy Angles by Young Dental make dental polishing more convenient because they have a small, round head and are designed to be particularly narrow, granting the therapist greater visibility and accessibility. The single-use products reduce the risk of infection and save time and costs as no sterilisation or preparation is required and the handpiece is less susceptible to wear and tear. The two-coloured DPAs in the Elite Cup and Petite Web Cup designs, either in straight design or with ergonomic 17 degree contrangle, help to reduce muscle fatigue.

The Young Dental Prophy Cups are made of special in-house rubber, offering greater flare, reducing splatter and helping achieve an excellent polishing result. The two-coloured cups come in various lengths and degrees of hardness, from firm to extra soft. They are free from latex and available with either a latch/RA or screw type attachment. Five different designs are offered, so that the user can meet patients' individual requirements. The Elite-Cup also has additional external ridges, which aid in the polishing of the interdental spaces. I The cups are individually blister packed to avoid cross-contamination.

www.youngdental.eu 